\title{
Interplay of the Inverse Proximity Effect and Magnetic Field in Out-of-Equilibrium Single-Electron Devices
}

\author{
Shuji Nakamura, ${ }^{1, *}$ Yuri A. Pashkin, ${ }^{2}$ Mathieu Taupin, ${ }^{3}$ Ville F. Maisi, ${ }^{4,5}$ Ivan M. Khaymovich,,${ }^{6,7}$, \\ Alexander S. Mel'nikov, ${ }^{7,8}$ Joonas T. Peltonen, ${ }^{4}$ Jukka P. Pekola, ${ }^{4}$ Yuma Okazaki, ${ }^{1}$ Satoshi Kashiwaya, ${ }^{1}$ \\ Shiro Kawabata, ${ }^{1}$ Andrey S. Vasenko, ${ }^{9}$ Jaw-Shen Tsai, ${ }^{10,11}$ and Nobu-Hisa Kaneko ${ }^{1}$ \\ ${ }^{1}$ National Institute of Advanced Industrial Science and Technology, \\ 1-1-1 Umezono, Tsukuba, Ibaraki 305-8563, Japan \\ ${ }^{2}$ Department of Physics, Lancaster University, Lancaster LA1 4YB, United Kingdom \\ ${ }^{3}$ Institute of Solid State Physics, Vienna University of Technology, \\ Wiedner Hauptstrasse 8-10, 1040 Vienna, Austria \\ ${ }^{4}$ Low Temperature Laboratory, Department of Applied Physics, Aalto University, 00076 Aalto, Finland \\ ${ }^{5}$ Center for Quantum Devices, Niels Bohr Institute, University of Copenhagen, \\ Universitetsparken 5, 2100 Copenhagen Ø, Denmark \\ ${ }^{6}$ Max Planck Institute for the Physics of Complex Systems, D-01187 Dresden, Germany \\ ${ }^{7}$ Institute for Physics of Microstructures, Russian Academy of Sciences, \\ 603950 Nizhny Novgorod, GSP-105, Russia \\ ${ }^{8}$ Lobachevsky State University of Nizhny Novgorod, 23 Prospekt Gagarina, \\ 603950 Nizhny Novgorod, Russia \\ ${ }^{9}$ National Research University Higher School of Economics, 101000 Moscow, Russia \\ ${ }^{10}$ RIKEN Center for Emergent Matter Science, Wako, Saitama 351-0198, Japan \\ ${ }^{11}$ Department of Physics, Tokyo University of Science, Kagurazaka, Tokyo 162-8601, Japan \\ (Received 13 December 2016; revised manuscript received 31 March 2017; published 26 May 2017)
}

\begin{abstract}
We show that a weak external magnetic field affects significantly nonequilibrium quasiparticle (QP) distributions under the conditions of the inverse proximity effect, using the single-electron hybrid turnstile as a generic example. Inverse proximity suppresses the superconducting gap in superconducting leads in the vicinity of turnstile junctions, thus, trapping hot QPs in this region. An external magnetic field creates additional QP traps in the leads in the form of vortices or regions with a reduced superconducting gap resulting in the release of QPs away from the junctions. We present clear experimental evidence of the interplay of the inverse proximity effect and magnetic field revealing itself in the superconducting gap enhancement and significant improvement of the turnstile characteristics. The observed interplay and its theoretical explanation in the context of QP overheating are important for various superconducting and hybrid nanoelectronic devices, which find applications in quantum computation, photon detection, and quantum metrology.
\end{abstract}

DOI: 10.1103/PhysRevApplied.7.054021

\section{INTRODUCTION}

The proximity effect induces superconducting correlations in a normal metal, which is in contact with a superconductor. In addition to playing an important role in the physics of superconductors [1], it also provides the basis for engineering the symmetry of the induced superconducting pairing in various hybrid structures [2-5]. Its counterpart, the inverse proximity effect, is responsible for the reduction of the superconducting order parameter due to the penetration of normal electrons into the superconductor [6]. Microscopically, both effects can be understood in terms of the Andreev reflection at the interface of a superconductor and a normal metal [7].

\footnotetext{
*shuji.nakamura@aist.go.jp

ivan.khaymovich@pks.mpg.de
}

The reduction of the superconducting gap caused by the inverse proximity effect creates traps for nonequilibrium quasiparticles (QPs) at the junction with a normal metal, which adversely affect the performance of many superconducting devices such as various types of photon detectors and bolometers (see, e.g., Refs. [8-11]), refrigerators based on normal-metal $(N)$-insulator $(I)$ - superconductor $(S)$ junctions [12], superconducting resonators [13], superconducting qubits $[14,15]$, and single-electron hybrid turnstiles [16]. Device performance degradation is especially significant for low-resistance devices since the noticeable gap reduction at the $S-I-N$ junction requires quite transparent interfaces. The problem of QP removal in these devices is usually solved by introducing additional QP traps away from the junction region, either by using normal-metal inclusions [17-19] or the local order-parameter suppression by an external magnetic field [12-16] or by using an alternative device design immune to QP overheating [20]. 
In this paper, we address an intriguing possibility to diminish the detrimental effect of the QP traps formed in the vicinity of the junctions by using an external magnetic field which leads to the nontrivial and counterintuitive increase of the superconducting gap in the junction areas. The presence of different trapping mechanisms and resulting redistribution of nonequilibrium QPs between the traps under the magnetic field is a rather general phenomenon, which reflects the fundamental properties of superconducting correlations and provides the means to control the gap profile in situ. The inhomogeneous gap profile and its sensitivity to the magnetic field can be used in superconducting and hybrid devices in which superconductors with different gap values are used [8,9] to tune the gap profile by the applied magnetic field. Indeed, in singlephoton detectors one needs to localize QPs generated by high-energy photons in the vicinity of the junction. However, to reduce the time when the detector is overheated and insensitive to subsequent photons, one needs to enhance QP relaxation. Therefore, the observed phenomenon of tunability of the gap profile in the magnetic field can be straightforwardly utilized in such devices. The magnetic-field-induced traps (e.g., vortex traps) for the considered gap engineering have also another advantage that is the perfect matching of superconducting parts with different gaps without the interface mismatch. Apparently, when controlling the position of the magnetic-field-induced traps (e.g., vortex traps), one should keep the distance from the junction to the nearest vortex to be at least 4 coherence lengths to avoid poisoning of the local density of states, thereby the low-energy states (see, e.g., Refs. [12-16,21]). The geometry of the superconducting leads is also important for the optimization problem due to its effect on both the quasiparticle diffusion [22] and vortex arrangement.

To observe the interplay between the magnetic field and inverse proximity effect experimentally, we study charge transport through a hybrid turnstile [23], i.e., a singleelectron transistor consisting of a normal-metal island with charging energy $E_{C}$ tunnel coupled to voltage-biased superconducting electrodes $(S-I-N-I-S)$ and controlled by both dc and rf gate voltages [see Fig. 1(a)]. By comparing the samples with different tunnel resistances $R_{T}$, we clearly demonstrate that the decrease of $R_{T}$ down to the order of the resistance quantum $R_{Q}=\left(h / e^{2}\right) \sim 25.8 \mathrm{k} \Omega$ enhances the excess current $\delta I=I-e f$ in the turnstile regime dominated by the hot QP contribution. Here, the product ef of the elementary electron charge $e$ and the drive frequency $f$ is the ideal value of the current $I$ in the turnstile regime. The decrease of $R_{T}$ also affects the superconducting gap $\Delta_{J}$ at the junctions $0<x<\ell$ keeping the gap $\Delta_{0}(x)=\Delta_{0}$ away from the junctions intact; see Fig. 1(b), where $x$ is the coordinate along the leads, and $\ell$ is the size of the proximized region at the $S-I-N$ junctions [Fig. 1(d)]. A weak magnetic field perpendicular to the sample reduces the turnstile excess current, and at the same (a)

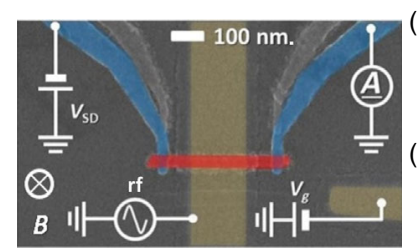

(b) $\Delta_{J}(0) \quad n_{\mathrm{QP}}(x)$

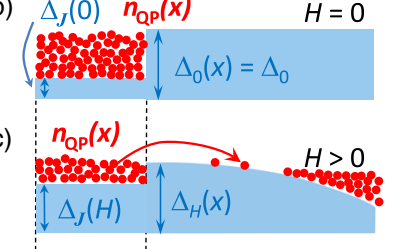

(d)

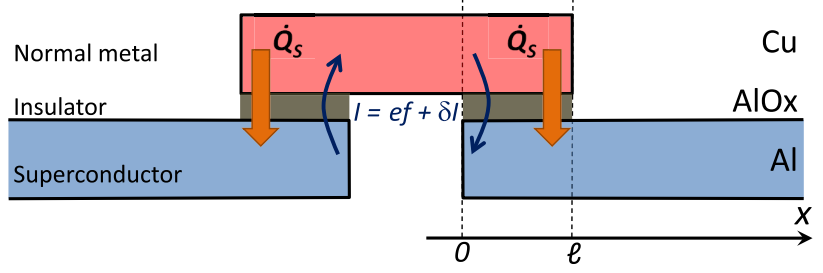

FIG. 1. (a) SEM image of the $S-I-N-I-S$ turnstile and measurement setup. False color identifies the superconducting Al leads (blue), the normal-metal $\mathrm{Cu}$ island (red), and the dc side-gate and rf bottom-gate electrodes (both yellow). (b),(c) The gap profile $\Delta_{H}(x)$ and $\Delta_{J}=\Delta_{H}(0)$ (blue shading) and the QP distribution $n_{\mathrm{QP}}(x)$ (red circles) in the leads at different magnetic field $H$ values. (d) Schematic cross section of the structure with charge (blue arrows) and heat (orange arrows) currents. In panels (b)-(d), the overlap junctions are located in the interval $0<x<\ell$ along the $x$ direction.

time, recovers $\Delta_{J}(H)$ closer to its bulk value $\Delta_{0}$. This observation is consistent with the developed theoretical model of the release of hot QPs from the vicinity of the junctions due to the reduction of the gap $\Delta_{H}(x)$ away from the junctions mediated by the magnetic field [see Fig. 1(c)] leading to the simultaneous gap increase at the junction. This model explains the magnetic field dependence of the excess current and gives semiquantitative agreement with the experimental data.

The rest of the paper is organized as follows. In Sec. II, we describe some details of the fabrication process and measurement setup and provide information about the sample characteristics. The main experimental results of the magnetic field effect on the turnstile operation and of the gap profile are given in Sec. III. Section IV is devoted to the theoretical analysis of the experimental data and discussion. Finally, in Sec. V we sum up our results and give an outlook. Some details of the derivation are given in the appendixes.

\section{FABRICATION, MEASUREMENT SETUP, AND SAMPLE CHARACTERIZATION}

The $S-I-N-I-S$ devices are fabricated on a thermally oxidized Si wafer with standard electron-beam lithography and metal deposition using the angle shadow evaporation technique [24]; see the SEM image of one of the samples in Fig. 1(a) and with a smaller magnification in Fig. 6 of Appendix B. Before the final fabrication step in which the $S-I-N-I-S$ structures are formed, the wafer goes through several processing steps to prepare the bonding pads and a ground plane. Each chip contains $\operatorname{Ti}(5 \mathrm{~nm}) / \mathrm{Au}(95 \mathrm{~nm})$ 
bonding pads and a $\operatorname{Ti}(5 \mathrm{~nm}) / \mathrm{Au}(50 \mathrm{~nm})$ ground plane that has a slot to accommodate a rf line. The rf line is extended to the center of the chip with a 30-nm-thick and 200-nm-wide Au strip. The whole wafer is then covered by a layer of $\mathrm{SiO}_{2}$ using spin-on glass, which is patterned to open the contact pads. Finally, we fabricate the $S-I-N-I-S$ turnstiles using a trilayer resist structure [copolymer resist $(400 \mathrm{~nm}) / \mathrm{Ge}(20 \mathrm{~nm}) /$ PMMA resist $(50 \mathrm{~nm})]$, which is formed by electron-beam lithography and dry etching. $S-I-N-I-S$ devices are connected to the bonding pads by $\mathrm{Al}$ leads stretching from the chip center to the bonding pads above the ground plane; thus, a large capacitance is formed between the dc leads and the ground plane protecting sensitive $S-I-N-I-S$ turnstiles from the electromagnetic noise penetrating into the sample package. $\mathrm{Al} / \mathrm{AlO}_{x} / \mathrm{Cu}$ tunnel junctions of the $S-I-N-I-S$ turnstiles are formed by the overlap of the 18-nm-thick Al leads and the 30-nmthick $\mathrm{Cu}$ island deposited in the e-gun evaporator at different angles through a suspended Ge mask formed by electron-beam lithography and dry etching. An aluminum oxide layer on the surface of the deposited $\mathrm{Al}$ film is grown by letting pure oxygen or $\mathrm{Ar}+\mathrm{O}_{2}$ mixture into the sample chamber. The tunnel junction resistance is controlled by varying the oxygen-argon pressure and oxidation time.

In this experiment, we use two different setups for oxidation and sample characterization (setup 1 and setup 2). Sample $H$ and sample $L$ are fabricated and measured in setup 1. Aluminum is oxidized under static conditions when a small amount of gas is introduced into the vacuum chamber. The oxidation conditions are $40 \mathrm{~s}$ under $37.5 \mathrm{mTorr}$ of pure oxygen for sample $H$, and 2 min under 97 mTorr $\mathrm{Ar}+\mathrm{O}_{2}(1 \%)$ for sample $L$. The measurement is performed in a homemade dilution refrigerator whose base temperature is around $100 \mathrm{mK}$. The measurement setup used in both setups is shown schematically in Fig. 1(a). The source-drain voltage $\left(V_{S D}\right)$ between the superconducting leads is applied using a commercial voltage source (SIM928), a dc gate-voltage $\left(V_{g}\right)$ tuning offset charges on the normal-metal island is applied using a commercial voltage source (SIM928), and the current is measured with a room-temperature current amplifier (DDPCA-300 from Femto) calibrated by a standard resistor and a calibrated voltage source at the Metrology Institute of Finland. The dc signals $\left(V_{S D}\right.$ and $\left.V_{g}\right)$ are filtered with Thermocoax cables, and the rf line has a $-20-\mathrm{dB}$ attenuator at $4.2 \mathrm{~K}$, thermalized in the helium bath, and a $-20-\mathrm{dB}$ attenuator at room temperature.

Sample $R$ is fabricated and measured in setup 2 . Aluminum is oxidized in a continuous gas-flow regime at a constant gas pressure maintained by an automatic pressure regulator. The oxidation condition is $2 \mathrm{~min}$ under $30 \mathrm{mTorr} \mathrm{Ar}+\mathrm{O}_{2}(10 \%)$. The measurement is performed in a commercial dilution refrigerator (Oxford Instruments Kelvinox 100) whose base temperature is also about
TABLE I. Parameters of the measured $S-I-N-I-S$ turnstiles.

\begin{tabular}{lccc}
\hline \hline Sample $^{\mathrm{a}}$ & $R_{T}(\mathrm{k} \Omega)$ & $E_{C} / \Delta_{0}$ & $\Delta_{0}(\mu \mathrm{eV})$ \\
\hline$H$ & 230 & 1.6 & 216 \\
$L$ & 55 & 1.6 & 215 \\
$R$ & 60 & 1.8 & 210 \\
\hline \hline
\end{tabular}

${ }^{\text {a }}$ Samples $H$ and $L$ are measured in setup 1, while sample $R$ is measured in setup 2. Setups 1 and 2 differ in sample shielding and rf wire filtering.

$100 \mathrm{mK}$. The voltage sources and the current amplifier are the same as those in setup 1. The calibration of the current amplifier is done at the Japanese Metrological Institute. The dc signals $\left(V_{S D}\right.$ and $\left.V_{g}\right)$ are filtered with Thermocoax cables and $\mathrm{Cu}$ powder filters, and the rf line has a low-pass filter (Mini-Circuits VLFX-1350), a Cu powder filter, and a $-40-\mathrm{dB}$ attenuator at room temperature.

The sample parameters $R_{T}, E_{C}$, and $\Delta_{0}$ of the three measured devices listed in Table I are extracted from the $I-V$ characteristics using standard numerical simulation based on Fermi's golden rule and the master equation [25]; see typical $I-V$ curves at different $V_{g}$ for sample $H$ in Figs. 2(a) and 2(b).

In both setups, the normal-metal island is capacitively coupled to the rf bottom-gate electrode isolated from the island by the $\mathrm{SiO}_{2}$ layer. In the turnstile experiments, an additional sinusoidal gate voltage with amplitude $A_{g}$ is applied to this rf gate electrode for the electronic pumping. The pumping experiments are carried out at a drive frequency $f=100 \mathrm{MHz}$ with the dc gate voltage $V_{g}$ fixed at the gate open state (the offset charge of the turnstile island is 0.5), and the source-drain voltage tuned to the optimal point $e V_{S D}=\Delta$ for the turnstile operation [23]. The current is measured with a room-temperature current amplifier calibrated by a standard resistor and a calibrated voltage source.
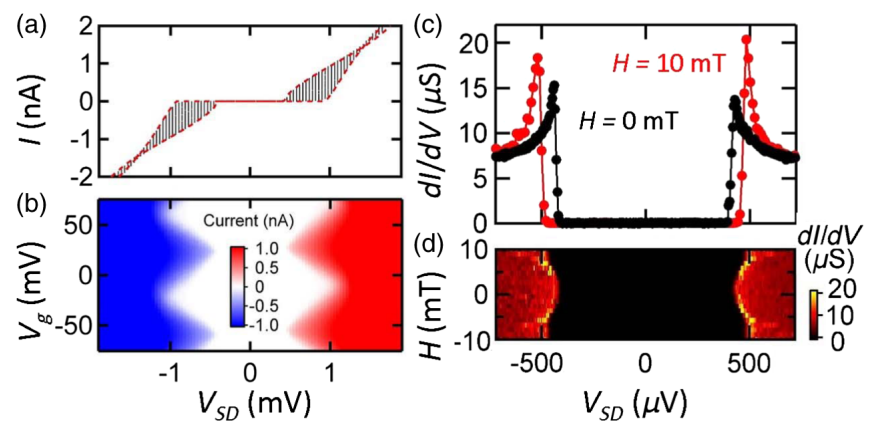

FIG. 2. (a) $I-V$ characteristics of sample $H$ for various dc gate voltages $V_{g}$. The dashed red lines are simulated $I-V$ characteristics for the gate open and closed states. (b) Source-drain turnstile current vs $V_{g}$ and $V_{S D}$ forming Coulomb blockade diamonds. (c) Differential conductance of sample $R$ as a function of bias voltage at zero (black) and finite (red) magnetic fields $H$. (d) Color plot of differential conductance vs $V_{S D}$ and $H$. 

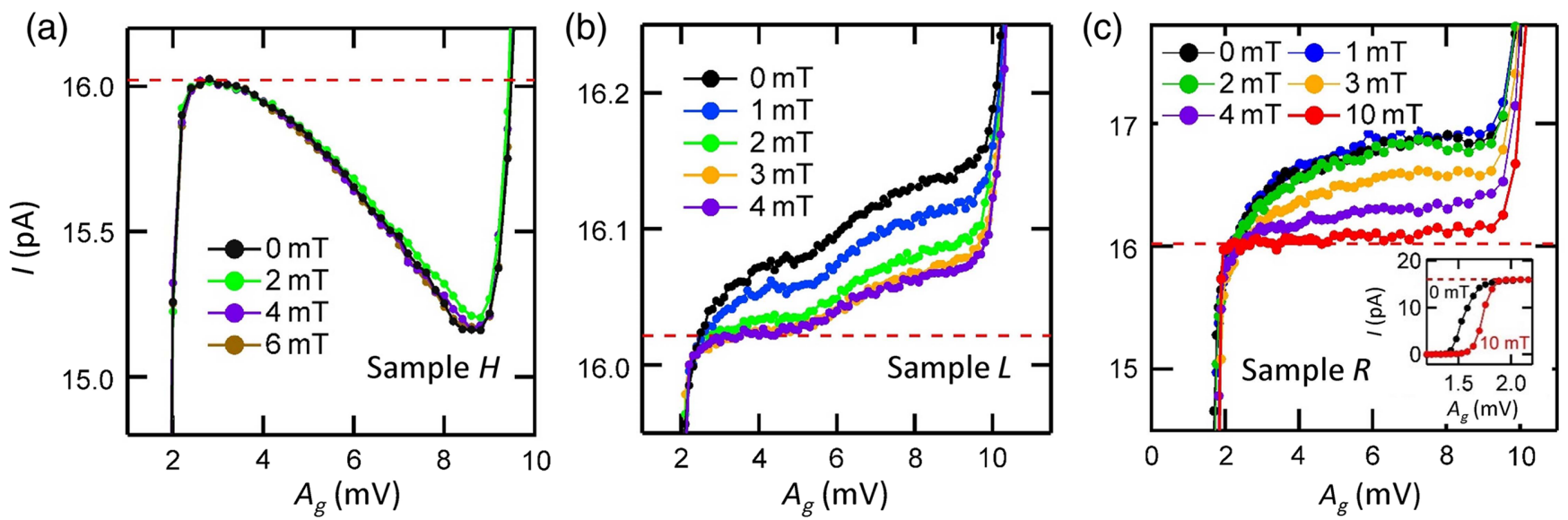

FIG. 3. Pumped current in the turnstile regime (colored dots) of (a) sample $H$, (b) sample $L$, and (c) sample $R$ vs rf gate amplitude at different magnetic fields. The horizontal red dashed lines show the ideal value of the pumped current $I=e f$ at the turnstile frequency $f=100 \mathrm{MHz}$. Panel (c) (inset) is a close-up of the onset of the current plateau at 0 and $10 \mathrm{mT}$.

\section{TURNSTILE MEASUREMENTS IN MAGNETIC FIELD}

At zero magnetic field, we observe that the turnstile current for high-resistance sample $H$ with $R_{T} \sim 9 R_{Q}$ [black dots in Fig. 3(a)] demonstrates backbending (red dashed line) at high $A_{g}$ values, in full agreement with the effect of backtunneling processes at high resistance and/or Coulomb energy [26]. Current $I$ in low-resistance samples $L$ and $R$ [black dots in Figs. 3(b) and 3(c)] with $R_{T} \sim 2 R_{Q}$ exceeds ef by $0.7 \%$ and $5 \%$, respectively.

In high-resistance sample $H$, a weak magnetic field applied perpendicular to the substrate has almost no effect on the turnstile current [color dots in Fig. 3(a)], whereas it reduces significantly the excess current in the lowresistance samples [color dots in Figs. 3(b) and 3(c)]. The
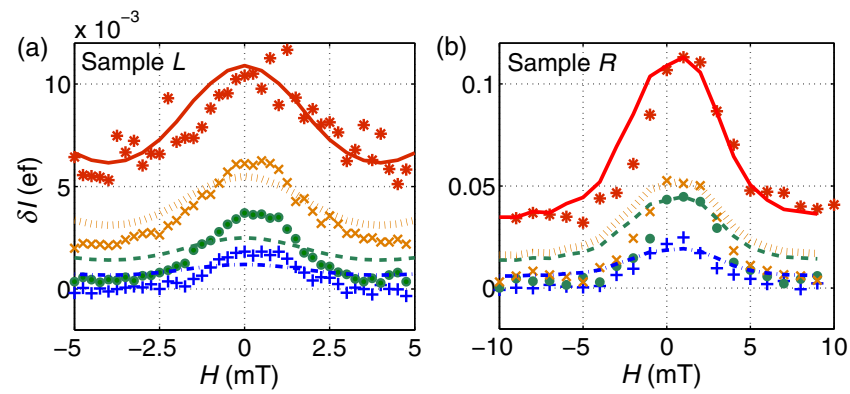

FIG. 4. The excess current $\delta I=I-e f$ normalized to ef through (a) sample $L$ and (b) sample $R$ vs magnetic field at $A_{g}=3,5,7,10 \mathrm{mV}$ (from bottom to top). The experimental data (symbols) are a cross section of the curves in Figs. 3(b) and 3(c) at fixed $A_{g}$. Theoretical curves (lines) are obtained using Eqs. (1) and (6). The zero-field gap $\Delta_{J}(0) / \Delta_{0}$ at the junctions normalized to its bulk value $\Delta_{0}$ is fitted as (a) 0.8 and (b) 0.6 for $A_{g}=10 \mathrm{mV}$ (red); the prefactor is taken to be $C=1$. Other theoretical curves are fitted with respect to $C\left(A_{g}\right)$ with values (a) $C=0.1,0.2,0.5$, 1 and (b) $C=0.17,0.40,0.47,1$ (from bottom to top). observed magnetic field dependence of the excess current for several gate amplitudes $A_{g}$ shown by the color symbols in Fig. 4 demonstrates this reduction both in samples $L$ and $R$. Together with the reduction of the excess current in samples $L$ and $R$, the rf threshold amplitude value $A_{g}^{\text {th }}=\Delta_{J} / e-$ $V_{S D} / 2$ increases [see inset of Fig. 3(c)] even though the dc source-drain and gate voltages are kept unchanged. This increase of $A_{g}^{\text {th }}$ points out that superconducting gap $\Delta_{J}$ at the junctions is enhanced by an applied magnetic field [27]. This increment of $\Delta_{J}(H)$ in the field is observed explicitly by measuring the differential conductance $d I / d V$ in the gate open state; see plots for sample $R$ in Figs. 2(c) and 2(d). The gap increases by more than $10 \%$ at $H=10 \mathrm{mT}$ relative to its zero-field value. The enhancement of the superconducting gap in sample $L$ is qualitatively the same (not shown).

\section{THEORETICAL MODEL AND COMPARISON WITH EXPERIMENTAL DATA}

Our quantitative theoretical description is based on the idea schematically shown in Figs. 1(b) and 1(c). At zero field, hot QPs produced due to the turnstile operation are trapped at the junction regions with reduced $\Delta_{J}$ [Fig. 1(b)] and cause the excess current [22]. Because of the lead geometry, magnetic field $H$ reduces the gap in the wide region away from the junctions (or even suppresses it due to vortex penetration) and opens the way for QPs to escape from the trap, therefore, diminishing the overheating of the proximized region [Fig. 1(c)]. The reduction of both overheating and the inverse proximity effect enhances the gap $\Delta_{J}$. Further increase of the field leads to the negative effect on $\Delta_{J}$ diminishing, therefore, the turnstile accuracy. In the theoretical model, we make the following assumptions: (i) The electron-electron relaxation rate is larger than the drive frequency and tunneling rates keeping electronic distributions in the leads to be of the Fermi-Dirac 
form with effective spatially dependent temperature $T(x)$ [28]. (ii) The excess current $\delta I=I-e f$ is dominated by the overheating contribution (see, e.g., Refs. [16,33,34])

$\delta I=C \frac{\sqrt{2 \pi \Delta_{J} k_{B} T_{J}}}{e R_{T}} \exp \left(-\frac{\Delta_{J}}{k_{B} T_{J}}\right) \equiv \frac{C \Delta_{J}}{e R_{T}} N_{\mathrm{QP}}(0)$,

with the numerical prefactor $C\left(A_{g}\right) \sim 1$ determined by the waveform and the amplitude $A_{g}$ of the rf gate voltage. (iii) In the proximized region $0<x<\ell$, the temperature $T(x)=T_{J}$, the gap $\Delta_{J}(H, x)=\Delta_{J}(H)$, and the normalized $\mathrm{QP}$ number $N_{\mathrm{QP}}(x)$ are constant [35]. (iv) Most of the dissipated heat $I \cdot V_{S D} \simeq 2 \dot{Q}_{S}$ goes to the leads and keeps the $N$ island close to equilibrium.

Because the lead width $w(x)$ increases gradually with the distance from the junction, the stationary temperature profile $T(x)$ averaged over $w(x)$ is determined by the solution of the quasi-one-dimensional heat-diffusion equation

$$
\frac{\partial}{\partial x}\left[w(x) \kappa_{S}(T(x), x) \frac{\partial}{\partial x} T(x)\right]=\dot{q}_{e-\mathrm{ph}}(T(x)) w(x)
$$

with the following boundary conditions at the junctions $0<x<\ell,\left.T\right|_{J}=T_{J}$, and away from it $x \rightarrow \infty$,

$$
\begin{aligned}
-\left.\kappa_{S}(T(x), x) \frac{\partial}{\partial x} T(x)\right|_{J} & =\dot{Q}_{S} / A, \\
\left.T(x)\right|_{x \rightarrow \infty} & =T_{0} .
\end{aligned}
$$

Here, $T_{0}$ is the phonon bath temperature, $\kappa_{S}(T(x), x) \sim$ $\left[2 \Delta_{H}^{2}(x) / e^{2} \rho_{n} T\right] e^{-\Delta_{H}(x) / k_{B} T}$ is the thermal conductivity of the superconductor, $A \simeq \ell w_{0}$ is the junction area, $\rho_{n}=$ $30 \mathrm{n} \Omega \mathrm{m}$ is the normal-state resistivity of the superconductor [22], $\dot{q}_{e \text {-ph }}$ is the density of the heat flux to the phonon bath.

Assuming the conservation of the heat flow and the temperature $T_{J}$ in the proximized region $0<x<\ell$ (iii) [36], one can solve Eq. (2) in the region $x>\ell$ with the boundary conditions analogous to Eqs. (3),

$$
\begin{gathered}
\left.\kappa_{S}(T, x) \frac{\partial}{\partial x} T(x)\right|_{x=\ell+0}=\dot{Q}_{\ell} / A_{\ell}, \\
\left.T(x)\right|_{x=\ell+0}=\left.T(x)\right|_{x=\ell-0} \equiv T_{J} .
\end{gathered}
$$

Here, $\dot{Q}_{\ell}=\dot{Q}_{S} A_{\ell} / A \simeq \dot{Q}_{S} d_{S} / w_{0}$ and $A_{\ell}=\ell d_{S}$ are the heat-flow rate and the cross-sectional area of the $S$ lead at $x=\ell$.

According to Refs. [16,22], at rather small temperatures $T_{0} \ll T \lesssim 0.2 \Delta_{H}(x) / k_{B}$, the density of the heat flux to the phonon bath can be approximated by the formula $\dot{q}_{e-\mathrm{ph}} \sim \Sigma T^{5} e^{-\Delta_{H}(x) / k_{B} T}$ neglecting the contributions proportional to $\propto e^{-2 \Delta_{H}(x) / k_{B} T}$ and $\propto e^{-\Delta_{H}(x) / k_{B} T_{0}}$. Here, $\Sigma \simeq$ $3 \times 10^{8} \mathrm{~W} \mathrm{~K}^{-5} \mathrm{~m}^{-3}$ is the electron-phonon material parameter [37-39].

In this limit, the main temperature dependence on both sides of the heat diffusion equation comes from the exponential $e^{-\Delta_{H}(x) / k_{B} T}$ and Eq. (2) at $x>\ell$ with logarithmic accuracy in terms of the $x$-dependent $\mathrm{QP}$ number $\mathcal{N}_{\mathrm{QP}}(x)$ takes the form

$$
\frac{\partial}{\partial x}\left[w(x) \frac{\partial}{\partial x} \mathcal{N}_{\mathrm{QP}}(x)\right]=L_{T}^{-2} \mathcal{N}_{\mathrm{QP}}(x) w(x),
$$

with the diffusion coefficient proportional to the width $w(x)$, and the weakly spatially dependent electron-phonon relaxation length $L_{T}^{-2}=e^{2} \rho_{n} k_{B} \Sigma T^{4}(x) / 2 \Delta_{H}(x)$. One can write the solution of this equation for $T_{J}$ and $N_{\mathrm{QP}}(\ell)$ (cf. [22,40])

$N_{\mathrm{QP}}(\ell)=\sqrt{\frac{2 \pi k_{B} T_{J}}{\Delta_{\ell}}} e^{-\Delta_{\ell} / k_{B} T_{J}} \simeq \frac{e^{2} \dot{Q}_{S} \rho_{n} \mathcal{R}_{H}[w(x)]}{\ell \sqrt{2 k_{B} T_{J} \Delta_{\ell}^{3} / \pi}}$,

with the superconducting gap just behind the junctions $\Delta_{\ell}=$ $\Delta_{H}(\ell)$ and the geometrical factor $\mathcal{R}_{H}[w(x)]$ defined as the ratio of the lead resistance normalized to the sheet resistance and being a functional of the lead width $w(x)$.

This normalized resistance $\mathcal{R}_{H}[w(x)]$ at zero magnetic field is the sum of the linear $l_{k} / w_{k}$ and logarithmic $\alpha^{-1} \ln \left(w_{k+1} / w_{k}\right)$ terms of one- and two-dimensional Green's functions of the Laplace equation for the $k$ th lead part of the length $l_{k}$ with constant width $w(x)=$ $w_{k-1}$ and the linearly increasing one $w(x)=w_{k-1}+$ $\delta x_{k}\left(w_{k}-w_{k-1}\right) / l_{k}$, respectively. Here, the coordinate $\delta x_{k}=x-x_{k}$ is shifted to be in the range $0<\delta x_{k}<l_{k}$ in the $k$ th lead part, and the opening angle is determined by $2 \tan \alpha=\left(w_{k}-w_{k-1}\right) / l_{k}$ (see Appendix B for the details of the sample geometry).

To estimate $\mathcal{R}_{H}$ at finite magnetic field, one should take into account the penetration of vortices into the lead at distances $x>x_{c}$, where the $S$ leads are already rather wide to let the first vortex enter $w\left(x_{c}\right) \sim \sqrt{\Phi_{0} / \pi H}$ [41]. As the electron-phonon relaxation term in vortices is of the order of the one in the normal metal [16], they simply relax the temperature $T(x) \simeq T_{0}$ to its phonon bath value $T_{0}$. Therefore, at distances $x>x_{c}$, one should truncate the summation in $\mathcal{R}_{H}$. Note that here we neglect the nonlocal contribution of vortices to the local density of states (LDOS) at the junction taken into account in Ref. [16] for the $N-I-S-I-N$ turnstile with a small superconducting island due to large $x_{c}>4 \xi$ compared to the coherence length $\xi$ [21].

In the limit of small excess current $\delta I \ll e f$, one can neglect its contribution to the heat-flux rate $\dot{Q}_{S} \simeq e f V_{S D} / 2$, and due to the exponential sensitivity of the left-hand side of Eq. (6) to $T_{J}$, one can disregard the polynomial $T_{J}$ dependence on the right-hand side. As a result, using the equality

$$
N_{\mathrm{QP}}(0)=\sqrt{\frac{2 \pi k_{B} T_{J}}{\Delta_{J}}}\left[N_{\mathrm{QP}}(\ell) \sqrt{\frac{\Delta_{\ell}}{2 \pi k_{B} T_{J}}}\right]^{\Delta_{J} / \Delta_{\ell}}
$$


with Eq. (6) substituted into it, the excess current normalized to ef (1) can be estimated as follows:

$$
\frac{\delta I(H)}{e f} \simeq \frac{C \sqrt{2 \pi k_{B} T_{J} \Delta_{J}}}{e^{2} R_{T} f}\left[\frac{e V_{S D}}{4 \Delta_{\ell}} \frac{e^{2} \rho_{n} f}{\ell k_{B} T_{J}} \mathcal{R}_{H}\right]^{\Delta_{J} / \Delta_{\ell}} .
$$

Because of the smallness of the term in the square brackets, the ratio $\Delta_{J}(H) / \Delta_{\ell}$ plays a significant role in the magnetic field dependence of $\delta I /(e f)$. The $H$ dependence of the terms in brackets is taken into account through the geometrical factor $\mathcal{R}_{H}$ which varies from $\mathcal{R}_{0} \simeq 35$ to $\mathcal{R}_{H} \simeq 20$ at $H=10 \mathrm{mT}$ due to the vortex penetration into the superconducting leads (see Appendix B for details).

The theoretical values of the excess current shown in Fig. 4 are calculated by substituting the self-consistent solution of Eq. (6) for $T_{J}$ into the general Eq. (1) with the fitting parameters $\Delta_{J}(0) / \Delta_{\ell}$ common for all $A_{g}$ and the amplitude-dependent factor $C\left(A_{g}\right)$; see Appendix A. The gap profile $\Delta_{J}(H) / \Delta_{J}(0)$ is taken from the experimental data; see Figs. 2(c) and 2(d). The numerical calculation reproduces the experimental results semiquantitatively for both samples $R$ and $L$. The data at the largest amplitude $A_{g}=10 \mathrm{mV}$ are fitted with $C\left(A_{g}\right)=1$. The deviation of the current from the theoretical curves at smaller amplitudes and at larger values of magnetic fields is possibly related to other contributions such as backtunneling, cotunneling, and Andreev processes as the quasiparticle contribution is suppressed. This effect is more significant in sample $L$ [Fig. 4(a)] where the excess current is approximately 10 times smaller. An order of magnitude difference in the amplitude and the different $A_{g}$ dependence of the excess current in samples $R$ and $L$ with close parameters (see Table I) could be related to the different experimental environment, namely, the sample-holder shielding and $\mathrm{rf}$ wire filtering in setups 1 and 2 causing the different profiles of the actual ac gate voltage applied to the sample.

The only subtle point not covered by the developed theoretical model is the $13 \%$ increase of the superconducting gap at the junctions at weak magnetic fields. Instead, the theory takes the magnetic field dependence of the gap from the experimental data and shows its evident relation with the excess current. This gap increase may, in principle, originate from the enhancement of the order parameter related to the hot quasiparticle density through the self-consistency equation. Another possible reason for the increase of $\Delta_{J}(H)$ is the absence of the $H$-mediated superfluid velocity in the dead end of the superconducting lead close to the junctions. However, according to the estimates (see Appendix $\mathrm{C}$ for details) and the numerical simulations of the Usadel equation, both of these effects cannot quantitatively explain the experimentally observed gap variation.

\section{CONCLUSIONS}

To conclude, we study single-electron pumping in the $S-I-N-I-S$ turnstile affected simultaneously by the inverse proximity effect and magnetic field. In the samples with a low junction resistance, a puzzling magnetic field dependence of the turnstile current and nonmonotonic magnetic field profile of the superconducting gap at the junctions are observed, while in the high-resistance sample, such effects are absent. This puzzle is resolved by the theoretical modeling taking into account both the inverse proximity effect leading to the quasiparticle trapping at the junctions and the overheating of the superconducting leads by hot quasiparticles resulting in the excess current. The perpendicular magnetic field releases hot quasiparticles from the proximized region by suppressing superconductivity away from the junctions and simultaneously weakening the proximity effect.

Our findings regarding the magnetic-field-induced gap increase can be particularly useful for improving the design of cryoelectronic devices suffering from hot quasiparticles. The observed effects of the inhomogeneous spatial gap profile and its tunability by magnetic field have a straightforward application to the devices benefiting from gap engineering, such as single-photon detectors and bolometers [8-11].

\section{ACKNOWLEDGMENTS}

We acknowledge fruitful discussions with Yoshitake Takane and Srinivas Gandrothula. This work is partially supported by KAKENHI, Grants No. 16H06090 (S. N.) and No. 15H03525 (Sh. K.), by the Academy of Finland, Projects No. 284594 and No. 272218 (M. T., J. T. P., and J.P.P.), by the UK EPSRC Grant No. EP/K01675X/1 (Yu. A. P.) and the Royal Society Grant No. WM110105 (Yu. A. P.), by the Russian Foundation for Basic Research and German Research Foundation (DFG) Grant No. KH 425/1-1 (I. M. K.), by the Russian Science Foundation Grant No. 17-12-01383 (A.S. M.), and by the project T3-97 "Macroscopic Quantum Phenomena at Low Temperatures" carried out within the framework of the Basic Research Program at the National Research University Higher School of Economics in 2016 (A. S. V.). We gratefully acknowledge the nanofabrication facilities of the Research Laboratory of NEC Corporation (Tsukuba, Japan) and the Otaniemi Research Infrastructure for Micro and Nanotechnologies (OtaNano, Finland) for the fabrication of the samples.

\section{APPENDIX A: QUASIPARTICLE-DOMINATED EXCESS CURRENT}

In this section, we verify whether the excess current $\delta I=$ $I-e f$ in the turnstile regime of a hybrid single-electron transistor, both of $N-I-S-I-N$ type with the superconducting island and of $S-I-N-I-S$ type with the normal-metal island, is dominated by the QP contribution to the tunneling rates $[33,34]$.

From the theoretical side as shown in the Supplemental Material 6 of Ref. [16], the excess current in both types of 

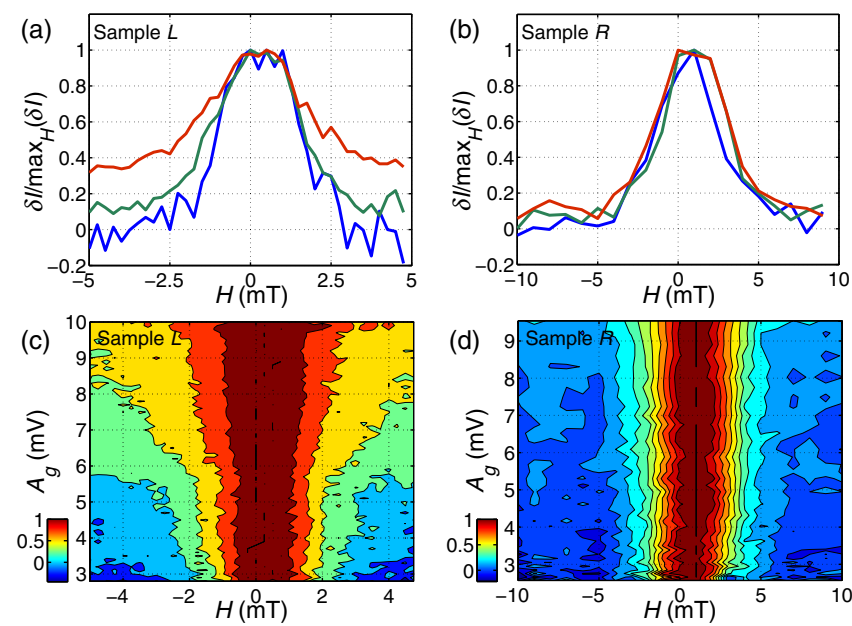

FIG. 5. The excess current $\delta I=I-e f$ in (a),(c) sample $L$ and (b),(d) sample $R$ normalized to its maximal value vs magnetic field $\max _{H}(\delta I)(\mathrm{a}),(\mathrm{b})$ for different amplitude values $A_{g}=3 \mathrm{mV}$ (blue), $5 \mathrm{mV}$ (green), and $7 \mathrm{mV}$ (red). Panels (c),(d) show color plots of $\delta I / \max _{H}(\delta I)$ at the turnstile plateau.

superconducting hybrid turnstiles takes the form of Eq. (1), where the overheating of and the inverse proximity effect in the $S$ parts is taken into account by the values of the superconducting gap $\Delta_{J}$ and the electronic temperature $T_{J}$ at the superconducting side close to the junction with the resistance $R_{T}$. A particular form of the numerical coefficient $C\left(A_{g}\right) \sim 1$ considered, e.g., in Ref. [16], depends on the waveform and the amplitude $A_{g}$ of the rf gate voltage and is considered as a fitting parameter of the model.

Other contributions to $\delta I$ such as Andreev tunneling, cotunneling, and Cooper-pair-electron cotunneling either do not depend on the drive amplitude, or their $A_{g}$ dependence cannot be factorized as in Eq. (1).

Therefore, to verify the dominant character of the QP contribution to the excess current experimentally, we normalize the magnetic-field-dependent data $\delta I\left(H, A_{g}\right)$ for different $A_{g}$ values to its zero-field value $\delta I\left(0, A_{g}\right)$; see Fig. 5. The figure shows that in sample $R$ (right panels), the excess current scales with the drive amplitude $A_{g}$ according to the theoretical factorizing formula (1), i.e., $\delta I\left(H, A_{g}\right)=C\left(A_{g}\right) \delta I_{0}(H)$. However, in sample $L$ (left panels), where the excess current is approximately 10 times smaller due to additional sample-holder shielding and wire filtering [see Fig. 4(a) in the main text], other contributions play an important role at larger values of magnetic fields as the QP contribution is suppressed. On one hand, this additional shielding leads to the smaller overheating effects [and, therefore, to the larger gap at the junction $\Delta_{J}(0)$ ], but on the other hand, this causes the lower quality of the fitting for sample $L$, as the QP current (1) is not the only contribution to the excess current in this case.

\section{APPENDIX B: DETAILS OF HEAT-DIFFUSION PROBLEM}

Here, we first verify assumption (iii) of the main text and then give the details of the solution of the heat-balance equation within the assumptions (i)-(iv) mentioned in the main text and of the calculation of the normalized resistance $\mathcal{R}_{H}[w(x)]$ for a certain space profile of the lead width $w(x)$ of the considered samples; see Fig. 6.

Starting with heat-diffusion equation (2) with boundary conditions (3), we first consider the proximized region $0<x<\ell$. As this region is affected homogeneously by the inverse proximity effect, we put $\Delta_{H}(x)=\Delta_{J}$, i.e., $\kappa_{J}[T(x)] \sim\left(2 \Delta_{J}^{2} / e^{2} \rho_{n} T(x)\right) e^{-\Delta_{J} / k_{B} T(x)}$ and neglect the electron-phonon relaxation $\dot{q}_{e \text {-ph }}$ due to the smallness of the region $\ell \ll L_{T}$ compared to the electron-phonon relaxation length $L_{T}$. These approximations lead to the conservation of the heat-flow rate in this region

$$
\dot{Q}_{\ell}=\dot{Q}_{S} A_{\ell} / A \simeq \dot{Q}_{S} d_{S} / w_{0},
$$

where $\dot{Q}_{\ell}$ and $A_{\ell}=\ell d_{S}$ are the heat-flow rate and the crosssectional area of the $S$ lead at $x=\ell$, and the width of the lead

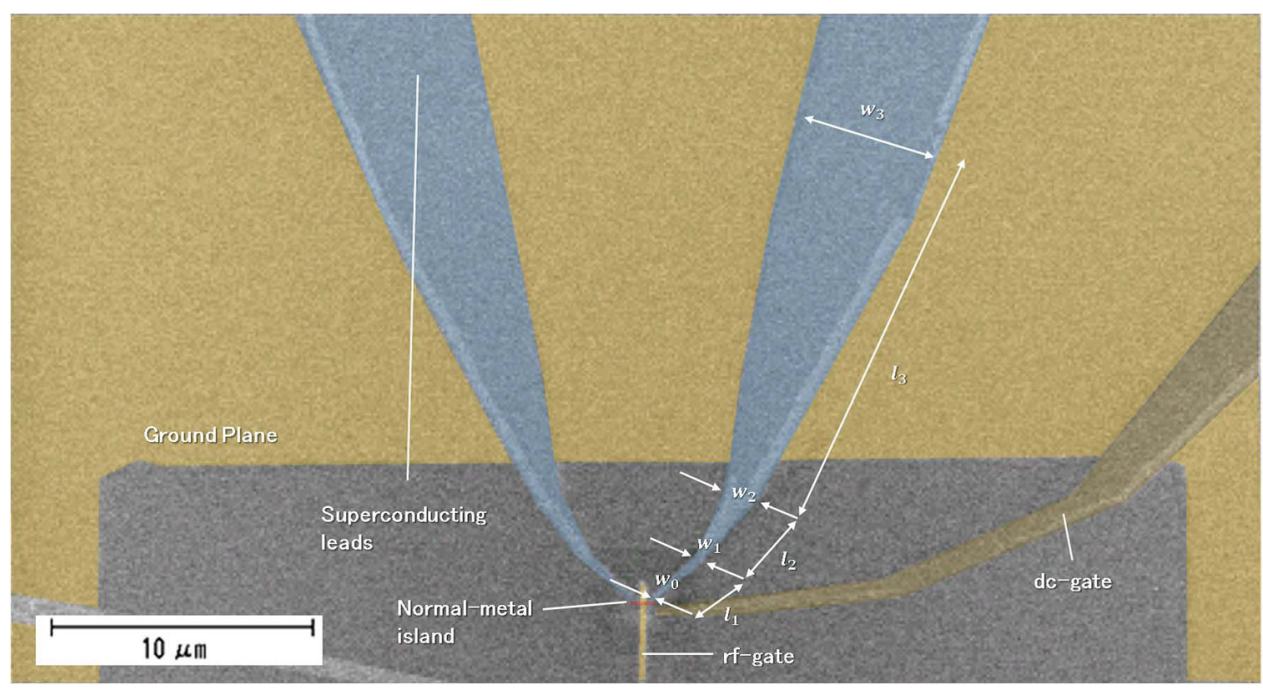

FIG. 6. SEM image of the $S-I-N-I-S$ single-electron transistor shown in Fig. 1(a) of the main text at larger spatial scale. As in the main text, false color identifies superconducting Al leads (blue), the normal-metal $\mathrm{Cu}$ island (red), and the ground plane as well as the dc side-gate and rf bottom-gate electrodes (all yellow). Widths $w_{k}$ and lengths $\ell, l_{k}$ mentioned in Eq. (B4) are shown in the figure. 
in this region is constant $w(0<x<\ell)=w_{0}$. As a result, the heat-diffusion equation at $0<x<\ell$ takes the form

$$
\begin{aligned}
\frac{\partial}{\partial x}\left[\kappa_{J}(T(x)) \frac{\partial}{\partial x} T(x)\right] & =0, \\
-\left.\kappa_{J}(T(x)) \frac{\partial}{\partial x} T(x)\right|_{x=0} & =\dot{Q}_{S} / A .
\end{aligned}
$$

We consider the heat injection to be concentrated at one end $x=0$, but as we show below, the detailed spatial distribution of the heat injection does not matter. Indeed, assuming the constant lead width in the region $x<\ell$, one can find that for $k_{B} T(x) \ll \Delta_{J}$, the QP number decays linearly with coordinate

$$
\mathcal{N}_{\mathrm{QP}}=\sqrt{\frac{2 \pi k_{B} T(x)}{\Delta_{J}}} e^{-\Delta_{J} / k_{B} T(x)} \simeq \mathcal{N}_{\mathrm{QP}}(0)-\frac{\dot{Q}_{S} e^{2} \rho_{n} x}{2 A k_{B} T_{J} \Delta_{J}} .
$$

In most cases, the second term is negligible provided the $x<\ell \ll L_{T}$; therefore, assumption (iii) of the main text $\mathcal{N}_{\mathrm{QP}} \simeq \mathcal{N}_{\mathrm{QP}}(0)$ and $T(x<\ell) \simeq T_{J}$ is valid.

In the rest of this section, we consider the concrete lead geometry of the measured samples (shown in Fig. 6) and give estimates for the corresponding geometrical factor $\mathcal{R}_{H}[w(x)]$ used in Eq. (6) and being a functional of the lead width profile. The lead geometry of the considered samples shown in Fig. 1 of the main text and in Fig. 6 leads to the following width profile:

$$
w(x)= \begin{cases}w_{0}+\frac{w_{1}-w_{0}}{l_{1}} \delta x_{0}, & 0<\delta x_{0}<l_{1}, \\ w_{1}+\frac{w_{2}-w_{1}}{l_{2}} \delta x_{1}, & 0<\delta x_{1}<l_{2}, \\ w_{2}+\frac{w_{3}-w_{2}}{l_{3}} \delta x_{2}, & 0<\delta x_{2}<l_{3}, \\ w_{3}, & 0<\delta x_{3},\end{cases}
$$

where $\delta x_{k}=x-\ell-\sum_{1}^{k} l_{k}$, and the junction region $0<x<\ell$ with $w(x)=w_{0}$ is followed by a set of linearly opening parts with corresponding lengths $l_{k}$ and width $w(x)$ changing from $w_{k-1}$ to $w_{k}$.

Taking the value estimates from the SEM micrographs $\ell \simeq w_{0} \simeq 50 \mathrm{~nm}, w_{1} \simeq 275 \mathrm{~nm}, w_{2} \simeq 842 \mathrm{~nm}, w_{3} \simeq 8.4 \mu \mathrm{m}$, $l_{1} \simeq 2.85 \mu \mathrm{m}, l_{2} \simeq 2.4 \mu \mathrm{m}, l_{3} \simeq 28 \mu \mathrm{m}$ and using the estimate $L_{T}^{2}=\left(R_{T} \ell^{2} d_{\mathrm{S}} / \rho_{n}\right) \sqrt{\left(2 k_{B} T / \pi \Delta_{\ell}\right)} \simeq(5 \mu \mathrm{m})^{2}$ from Ref. [22] (in the presence of a normal shadow trap lying on top of the $S$ lead far away from the junction with the same sheet $N-I-S$ resistance), one can obtain at zero magnetic field

$$
\mathcal{R}_{0}=\sum_{k=1}^{3} \frac{l_{k}}{w_{k}-w_{k-1}} \ln \left(\frac{w_{k}}{w_{k-1}}\right)+\frac{L_{T}}{w_{3}} \simeq 35
$$

Taking into account the estimate of $\mathcal{R}_{H}$ at finite magnetic field given in the main text with the critical width for the first vortex entry $w\left(x_{c}\right) \simeq \sqrt{\Phi_{0} / \pi H} \simeq$ $250 \mathrm{~nm}$ for $H \simeq 10 \mathrm{mT}$, we keep only the first term in sum in Eq. (B5) with $w_{1}$ substituted by $w\left(x_{c}\right)$ in the logarithm. It gives eventually

$$
\mathcal{R}_{H} \approx \frac{l_{1}}{w_{1}-w_{0}} \ln \left[\frac{w\left(x_{c}\right)}{w_{0}}\right] \simeq 20 .
$$

\section{APPENDIX C: ESTIMATES OF THE GAP $\Delta_{J}$ VARIATIONS WITH $H$ AND $T_{J}$}

According to the experimental data [see Fig. 2(c) of the main text], the gap $\Delta_{J}$ at the junction of sample $R$ increases by $13 \%$ as the magnetic field increases from 0 to $10 \mathrm{mT}$ [42]. In this section, we estimate the influence of a depairing parameter and of QP traps (via the variation of the electronic temperature $T_{J}$ at the junction) on $\Delta_{J}$ and show that these effects are negligible in the measured experimental setup.

We start with the depairing parameter effect. In the diffusion limit with coherence length $\xi \gg \mathfrak{l}_{\text {MFP }}$ well exceeding the mean free path $\mathfrak{l}_{\mathrm{MFP}}$, one can find the superconducting gap $\Delta_{J}$ in the QP spectrum at a finite magnetic field $H$ as follows (see, e.g., Refs. [43-46]):

$$
\Delta_{J}=\Delta_{S}(H)\left(1-\gamma_{H}^{2 / 3}\right)^{3 / 2},
$$

where $\Delta_{S}(H)=\Delta_{0} \exp \left(-\pi \gamma_{H} / 4\right)$ is the superconducting order parameter, $\gamma_{H}=\left[\hbar\left\langle v_{S}^{2}\right\rangle / 2 D \Delta_{S}(H)\right]$ is the depairing parameter, $D_{S}$ is the diffusion coefficient in the superconductor, and $\left\langle v_{S}^{2}\right\rangle=(\pi D H \ell)^{2} / 3 \Phi_{0}^{2}$ is the averaged square of the superfluid velocity.

Strictly speaking, this analysis works for small widths $\ell \ll \xi$ of the superconducting leads near the junction; therefore, we use it only for estimates. For experimental parameters $\xi \simeq 100 \mathrm{~nm}, \ell=50 \mathrm{~nm}$, and $H \simeq 10 \mathrm{mT}$, the depairing parameter is rather small

$$
\gamma_{H}=\frac{1}{6}\left(\frac{\pi H \xi \ell}{\Phi_{0}}\right)^{2} \simeq 0.001,
$$

and it leads to a decrease of the superconducting gap by less than $2 \%$,

$$
1-\Delta_{J}(H) / \Delta_{0} \simeq 0.016,
$$

which is nearly an order of magnitude smaller than the experimentally observed increase by $5 \%$ and $13 \%$ for samples $L$ and $R$, respectively.

Now we consider the effect of QP traps on the gap $\Delta_{J}$. Starting with the experimental data for the excess current $\delta I /(e f)$ shown in Fig. 4 of the main text and taking into account Eq. (1), drive frequency $f=100 \mathrm{MHz}$, and junction resistance $R_{T} \simeq 55-60 \mathrm{k} \Omega$, we estimate the ratio $r(H)=k_{B} T_{J}(H) / \Delta_{J}(H)$ for both samples $L$ and $R$ at $H_{0}=0$ and $H_{1}=10 \mathrm{mT}$; see Table II.

As the superconducting gap at the junction can be only reduced relatively to its equilibrium bulk value $\Delta_{0}$ both by 
TABLE II. Temperature-to-gap ratio.

\begin{tabular}{lcc}
\hline \hline Sample & $k_{B} T_{J}(0) / \Delta_{J}(0)$ & $k_{B} T_{J}\left(H_{1}\right) / \Delta_{J}\left(H_{1}\right)$ \\
\hline$L$ & 0.094 & 0.086 \\
$R$ & 0.120 & 0.095 \\
\hline \hline
\end{tabular}

the inverse proximity effect and by the depairing parameter (see estimates above), one can use the ratios mentioned in Table II as the upper bounds for temperatures $k_{B} T_{J}(H) \leq$ $r(H) \Delta_{0}$. On the other hand, any variations of the electronic temperature $T_{J}(H)$ (due to the presence of QP traps away from junctions) at such small absolute values $\left[k_{B} T_{J}(H) \leq\right.$ $0.12 \Delta_{0}$ ] can only slightly change the self-consistent orderparameter value

$$
\begin{aligned}
1-\frac{\Delta_{S}\left(H, T_{J}(H)\right)}{\Delta_{0}} & \simeq \sqrt{\frac{2 \pi k_{B} T_{J}(H)}{\Delta_{S}(H)}} e^{-\Delta_{S}(H) / k_{B} T_{J}(H)} \\
& \lesssim \frac{e R_{T} \delta I}{\Delta_{0}}<2 \times 10^{-4}
\end{aligned}
$$

Here we used the maximal value of $\delta I /(e f) \simeq 0.12$ for sample $R$ on the plateau.

We also perform numerical calculations of the LDOS by solving the Usadel equation together with the selfconsistency equation for a superconducting order parameter in a quasi-one-dimensional approximation of the leads near the junction and in the overlap geometry of a $\mathrm{N}-\mathrm{I}-\mathrm{S}$ tunnel contact used in the experiments [see Fig. 1(a) of the main text]. We do not present the results of these numerical simulations here, as it is shown that both the negative effect of the depairing parameter and the stimulating effect of the QP traps are in quantitative agreement with the simple estimates that we mention above and cannot explain the experimentally observed increase of the superconducting gap in a weak magnetic field.

[1] P. G. de Gennes, Superconductivity of Metals and Alloys (Addison-Wesley, New York, 1989).

[2] V. Mourik, K. Zuo, S. M. Frolov, S. R. Plissard, E. P. A. M. Bakker, and L. P. Kouwenhoven, Signatures of Majorana fermions in hybrid superconductor-semiconductor nanowire devices, Science 336, 1003 (2012).

[3] M. Eschrig and T. Löfwander, Triplet supercurrents in clean and disordered half-metallic ferromagnets, Nat. Phys. 4, 138 (2008).

[4] F. Giazotto, J. T. Peltonen, M. Meschke, and J. P. Pekola, Superconducting quantum interference proximity transistor, Nat. Phys. 6, 254 (2010).

[5] A. Ronzani, C. Altimiras, and F. Giazotto, Highly Sensitive Superconducting Quantum-Interference Proximity Transistor, Phys. Rev. Applied 2, 024005 (2014).

[6] P. G. D. Gennes, Boundary effects in superconductors, Rev. Mod. Phys. 36, 225 (1964).
[7] M. Tinkham, Introduction to Superconductivity, 2nd ed. (Dover Publications, New York, 2004).

[8] A. A. Golubov and E. P. Houwman, Quasiparticle relaxation rates in a spatially inhomogeneous superconductor, Physica (Amsterdam) 205C, 147 (1993).

[9] A. A. Golubov, E. P. Houwman, J. G. Gijsbertsen, J. Flokstra, H. Rogalla, J. B. le Grand, and P. A. J. de Korte, Quasiparticle lifetimes and tunneling times in a superconductor-insulatorsuperconductor tunnel junction with spatially inhomogeneous electrodes, Phys. Rev. B 49, 12953 (1994).

[10] G. N. Goltsman, O. Okunev, G. Chulkova, A. Lipatov, A. Semenov, K. Smirnov, B. Voronov, and A. Dzardanov, Picosecond superconducting single-photon optical detector, Appl. Phys. Lett. 79, 705 (2001).

[11] P. L. Richards, Bolometers for infrared and millimeter waves, J. Appl. Phys. 76, 1 (1994).

[12] J. T. Peltonen, J. T. Muhonen, M. Meschke, N. B. Kopnin, and J. P. Pekola, Magnetic-field-induced stabilization of nonequilibrium superconductivity in a normal-metal/insulator/ superconductor junction, Phys. Rev. B 84, 220502(R) (2011).

[13] I. Nsanzineza and B. L. T. Plourde, Trapping a Single Vortex and Reducing Quasiparticles in a Superconducting Resonator, Phys. Rev. Lett. 113, 117002 (2014).

[14] C. Wang, Y. Y. Gao, I. M. Pop, U. Vool, C. Axline, T. Brecht, R. W. Heeres, L. Frunzio, M. H. Devoret, G. Catelani, L. I. Glazman, and R. J. Schoelkopf, Measurement and control of quasiparticle dynamics in a superconducting qubit, Nat. Commun. 5, 5836 (2014).

[15] U. Vool, I. M. Pop, K. Sliwa, B. Abdo, C. Wang, T. Brecht, Y. Y. Gao, S. Shankar, M. Hatridge, G. Catelani, M. Mirrahimi, L. Frunzio, R. J. Schoelkopf, L. I. Glazman, and M. H. Devoret, Non-Poissonian Quantum Jumps of a Fluxonium Qubit Due to Quasiparticle Excitations, Phys. Rev. Lett. 113, 247001 (2014).

[16] M. Taupin, I. M. Khaymovich, M. Meschke, A. S. Mel'nikov, and J. P. Pekola, Tunable quasiparticle trapping in Meissner and vortex states of mesoscopic superconductors, Nat. Commun. 7, 10977 (2016).

[17] D. J. Goldie, N. E. Booth, C. Patel, and G. L. Salmon, Quasiparticle Trapping from a Single-Crystal Superconductor into a Normal-Metal Film via the Proximity Effect, Phys. Rev. Lett. 64, 954 (1990).

[18] J. N. Ullom, P. A. Fisher, and M. Nahum, Measurements of quasiparticle thermalization in a normal metal, Phys. Rev. B 61, 14839 (2000).

[19] S. Rajauria, L. M. A. Pascal, Ph. Gandit, F. W. J. Hekking, B. Pannetier, and H. Courtois, Efficiency of quasiparticle evacuation in superconducting devices, Phys. Rev. B 85, 020505(R) (2012).

[20] D. M. T. van Zanten, D. M. Basko, I. M. Khaymovich, J. P. Pekola, H. Courtois, and C. B. Winkelmann, Single Quantum Level Electron Turnstile, Phys. Rev. Lett. 116, 166801 (2016).

[21] J. T. Peltonen, P. Virtanen, M. Meschke, J. V. Koski, T. T. Heikkilä, and J. P. Pekola, Thermal Conductance by the Inverse Proximity Effect in a Superconductor, Phys. Rev. Lett. 105, 097004 (2010).

[22] H. S. Knowles, V.F. Maisi, and J. P. Pekola, Probing quasiparticle excitations in a hybrid single electron transistor, Appl. Phys. Lett. 100, 262601 (2012). 
[23] J. P. Pekola, J. J. Vartiainen, M. Möttönen, O.-P. Saira, M. Meschke, and D. V. Averin, Hybrid single-electron transistor as a source of quantized electric current, Nat. Phys. 4, 120 (2008).

[24] T. A. Fulton and G. J. Dolan, Observation of SingleElectron Charging Effects in Small Tunnel Junctions, Phys. Rev. Lett. 59, 109 (1987).

[25] A. Kemppinen, M. Meschke, M. Möttönen, D. V. Averin, and J. P. Pekola, Quantized current of a hybrid singleelectron transistor with superconducting leads and a normal-metal island, Eur. Phys. J. Spec. Top. 172, 311 (2009).

[26] A. Kemppinen, S. Kafanov, Y. A. Pashkin, J. S. Tsai, D. V. Averin, and J.P. Pekola, Experimental investigation of hybrid single-electron turnstiles with high charging energy, Appl. Phys. Lett. 94, 172108 (2009).

[27] A similar effect of the increase of a threshold in a NIS cooler associated with the superconducting gap has been observed in Ref. [12].

[28] Here we consider only the quasiequilibrium regime with the Fermi-Dirac electron distribution function $f(E)$ in contrast to several works [29-32] considering the general form of $f(E)$ both in the superconducting and normal parts.

[29] J. P. Pekola, T. T. Heikkilä, A. M. Savin, J. T. Flyktman, F. Giazotto, and F. W. J. Hekking, Limitations in Cooling Electrons Using Normal-Metal-Superconductor Tunnel Junctions, Phys. Rev. Lett. 92, 056804 (2004).

[30] F. Giazotto, T. T. Heikkilä, F. Taddei, Rosario Fazio, J. P. Pekola, and F. Beltram, Tailoring Josephson Coupling through Superconductivity-Induced Nonequilibrium, Phys. Rev. Lett. 92, 137001 (2004).

[31] M. A. Laakso, P. Virtanen, F. Giazotto, and T. T. Heikkilä, Nonequilibrium characteristics in all-superconducting tunnel structures, Phys. Rev. B 75, 094507 (2007).

[32] I. M. Khaymovich and D. M. Basko, Recovery of $S-I-N-I-S$ turnstile accuracy in a strongly nonequilibrium regime, Phys. Rev. B 94, 165158 (2016).
[33] O.-P. Saira, A. Kemppinen, V. F. Maisi, and J. P. Pekola, Vanishing quasiparticle density in a hybrid $\mathrm{Al} / \mathrm{Cu} / \mathrm{Al}$ singleelectron transistor, Phys. Rev. B 85, 012504 (2012).

[34] V. F. Maisi, Ph.D. thesis, Aalto University, 2014.

[35] This approximation is valid due to the homogeneity of the heat inflow and the smallness of $\ell$ with respect to the electron-phonon relaxation length $L_{T}$.

[36] This assumption is verified in Appendix B.

[37] R. L. Kautz, G. Zimmerli, and J. M. Martinis, Self-heating in the Coulomb-blockade electrometer, J. Appl. Phys. 73, 2386 (1993).

[38] F. Giazotto, T. T. Heikkilä, A. Luukanen, A. M. Savin, and J. P. Pekola, Opportunities for mesoscopics in thermometry and refrigeration: Physics and applications, Rev. Mod. Phys. 78, 217 (2006).

[39] V. F. Maisi, S. V. Lotkhov, A. Kemppinen, A. Heimes, J. T. Muhonen, and J. P. Pekola, Excitation of Single Quasiparticles in a Small Superconducting Al Island Connected to Normal-Metal Leads by Tunnel Junctions, Phys. Rev. Lett. 111, 147001 (2013).

[40] S. Rajauria, H. Courtois, and B. Pannetier, Quasiparticlediffusion-based heating in superconductor tunneling microcoolers, Phys. Rev. B 80, 214521 (2009).

[41] G. Stan, S. B. Field, and J. M. Martinis, Critical Field for Complete Vortex Expulsion from Narrow Superconducting Strips, Phys. Rev. Lett. 92, 097003 (2004).

[42] The corresponding increase of the gap at the junction of sample $L$ is approximately $5 \%$.

[43] S. Skalski, O. Betbeder-Matibet, and P. R. Weiss, Properties of superconducting alloys containing paramagnetic impurities, Phys. Rev. 136, A1500 (1964).

[44] P. Fulde, Tunneling density of states for a superconductor carrying a current, Phys. Rev. 137, A783 (1965).

[45] K. Maki and P. Fulde, Equivalence of different pair-breaking mechanisms in superonductors, Phys. Rev. 140, A1586 (1965).

[46] A. Anthore, H. Pothier, and D. Esteve, Density of States in a Superconductor Carrying a Supercurrent, Phys. Rev. Lett. 90, 127001 (2003). 